University of Wollongong

Research Online

Faculty of Engineering and Information

Faculty of Engineering and Information

Sciences - Papers: Part B

Sciences

2017

\title{
Leveraging Stacked Denoising Autoencoder in Prediction of Pathogen-Host Protein-Protein Interactions
}

Huaming Chen

University of Wollongong, hc007@uowmail.edu.au

Jun Shen

University of Wollongong, jshen@uow.edu.au

Lei Wang

University of Wollongong, leiw@uow.edu.au

Jiangning Song

Monash University, jiangning.song@monash.edu

Follow this and additional works at: https://ro.uow.edu.au/eispapers1

Part of the Engineering Commons, and the Science and Technology Studies Commons

Research Online is the open access institutional repository for the University of Wollongong. For further information contact the UOW Library: research-pubs@uow.edu.au 


\title{
Leveraging Stacked Denoising Autoencoder in Prediction of Pathogen-Host Protein-Protein Interactions
}

\begin{abstract}
In big data research related to bioinformatics, one of the most critical areas is proteomics. In this paper, we focus on the protein-protein interactions, especially on pathogen-host protein-protein interactions (PHPPIs), which reveals the critical molecular process in biology. Conventionally, biologists apply in-lab methods, including small-scale biochemical, biophysical, genetic experiments and large-scale experiment methods (e.g. yeast-two-hybrid analysis), to identify the interactions. These in-lab methods are time consuming and labor intensive. Since the interactions between proteins from different species play very critical roles for both the infectious diseases and drug design, the motivation behind this study is to provide a basic framework for biologists, which is based on big data analytics and deep learning models. Our work contributes in leveraging unsupervised learning model, in which we focus on stacked denoising autoencoders, to achieve a more efficient prediction performance on PHPPI. In this paper, we further detail the framework based on unsupervised learning model for PHPPI researches, while curating a large imbalanced PHPPI dataset. Our model demonstrates a better result with the unsupervised learning model on PHPPI dataset.
\end{abstract}

\section{Keywords}

protein-protein, leveraging, interactions, stacked, denoising, autoencoder, prediction, pathogen-host

\section{Disciplines}

Engineering | Science and Technology Studies

\section{Publication Details}

Chen, H., Shen, J., Wang, L. \& Song, J. (2017). Leveraging Stacked Denoising Autoencoder in Prediction of Pathogen-Host Protein-Protein Interactions. IEEE 6th International Congress on Big Data (pp. 368-375). United States: IEEE. 


\section{Leveraging Stacked Denoising Autoencoder in Prediction of Pathogen-Host Protein-Protein Interactions}

\author{
Huaming Chen, Jun Shen, Lei Wang \\ School of Computing and Information Technology \\ University of Wollongong \\ Wollongong, NSW, Australia \\ Email:hc007@uowmail.edu.au,\{jshen,leiw\}@uow.edu.au
}

\author{
Jiangning Song \\ Department of Biochemistry and Molecular Biology \\ Monash University \\ Melbourne, Victoria, Australia \\ Email: Jiangning.Song@monash.edu
}

\begin{abstract}
In big data research related to bioinformatics, one of the most critical areas is proteomics. In this paper, we focus on the protein-protein interactions, especially on pathogen-host protein-protein interactions (PHPPIs), which reveals the critical molecular process in biology. Conventionally, biologists apply in-lab methods, including small-scale biochemical, biophysical, genetic experiments and large-scale experiment methods (e.g. yeast-two-hybrid analysis), to identify the interactions. These in-lab methods are time consuming and labor intensive. Since the interactions between proteins from different species play very critical roles for both the infectious diseases and drug design, the motivation behind this study is to provide a basic framework for biologists, which is based on big data analytics and deep learning models. Our work contributes in leveraging unsupervised learning model, in which we focus on stacked denoising autoencoders, to achieve a more efficient prediction performance on PHPPI. In this paper, we further detail the framework based on unsupervised learning model for PHPPI researches, while curating a large imbalanced PHPPI dataset. Our model demonstrates a better result with the unsupervised learning model on PHPPI dataset.
\end{abstract}

Keywords-big data; PHPPI; denoising autoencoder; prediction; machine learning

\section{INTRODUCTION}

Immersed in many disciplines, including computer vision, online resources, health care, urban city and bioinformatics study, big data analytics technology is influencing every area of our life with the enormously available data [1-4]. The big data is considered as a data-driven research area in combination with data mining and machine learning technologies, so the verified and well represented data are crucial. Through decades of efforts by the biologists, tremendous datasets are being recorded in bioinformatics. Nowadays the biologists and computer science researchers are working together to tackle the challenges in knowledge discovery by building computational models in both proteomics and genomics disciplines [5, 6].

In our study, we focus on the proteomics area. Proteomics research draws a lot of attention due to the huge emerging 'omics' data in recent years. These data are accumulated in an extraordinary speed benefiting from high throughput technologies. Identifying protein-protein interactions (PPI) with a comprehensive understanding is essential for the studies of biological functions, which are fundamental to particular research, like drug design and infectious diseases. Among these PPI research, the computational studies for PPI mostly focused on 'intra-species' PPI, which mainly concerned the interactions between two proteins from the same species.

Since identifying PPIs is essential for understanding the whole biological functions and most diseases occurring between the hosts and pathogens, we focus on the interactions between proteins from two different species, namely 'interspecies' PPI. As pathogen-host PPI plays an important role in both the mechanisms of infection and medicine treatment, obtaining a better understanding and prediction of PHPPI will bring huge benefits for biologists.

On the other hand, deep learning is currently booming and it has achieved a lot of inspiring research outcomes [7]. It is considered as the most effective model with its ability to generate higher level representation features for model learning, when there is sufficient data [8-10]. Several popular network models are being widely used, including convolutional neural network $(\mathrm{CNN})$ and deep belief net (DBN).

However, the literature research mostly focus on relatively small datasets with balanced ratio. Currently, there is little research on PHPPI, especially while PHPPI datasets appear to have two important characteristics, which normally exhibit high skewed ratio and large data size, as reported in our earlier study [11]. Herein we propose to leverage unsupervised learning model, especially the stacked denoising autoencoders (SdA) in our model, for PHPPI prediction. A general framework with unsupervised learning model is further built to tackle the unique challenges from PHPPI research, which need to deal with large data size and imbalanced ratio between binary classes. Inside this framework, we anticipate that $\mathrm{SdA}$, as the primary unsupervised learning model, could automatically represent higher level features for further model classifier. In our experiments, We observed that the higher level representation by unsupervised learning 
could be the key to a better performance for prediction in PHPPI. We demonstrate this observation by deploying our framework to two selected pathogen species. These two pathogen species are 'Francisella tularensis' and 'Clostridium difficile', while the host is human.

The rest of this paper is organized as follows: Section II reviews the related work; Section III presents our framework with deep learning methods; Section IV gives our detailed PHPPI datasets and the evaluation metrics; Section V is our result analysis and discussion. Finally we conclude this paper and point out future work in Section VI.

\section{RELATED WORK}

More and more experiments are conducted to verify whether a pair of proteins interact with each other. Among these, only the positive PPIs, which means a pair of proteins has interacting relationship, are manually recorded. However, identifying these positive PPIs could be time consuming and labor intensive in biological experiments, which could also introduce high false positive rate. The uncertainty of experimental results for detection of interactions between host and pathogen proteins is one of the main obstacles for biologists [12]. How to provide highly confident prediction for further experimental verification is our goal in this study.

Yeast and human are considered as two special species since most of PPI research focus on these 'intra-species' PPIs. [13] gave a detailed and insightful analysis on yeast protein interactions, while [14] proposed an algorithm called PrePPI to yield over 30,000 high-confidence interactions for yeast and over 300,000 interactions for human. This algorithm proposed to utilize Bayesian statistics to predict interactions with 3D structural information. [15] presented a novel sequences feature representation method of proteins to achieve a better result than other works. The PPI applied in [15] were extracted from the yeast protein-protein interactions.

Among these research a balanced dataset was normally applied, which could be either from the available websites or built by researchers themselves. The balanced ratio between the positive and negative PPI data is nearly $1: 1$. For PHPPI, the ratio between positive and negative pairs is highly skewed, which is normally 1:100 to $1: 500$. However, the ratio $1: 100$ is generally considered to build a less-biased classifier for prediction $[11,16,17]$.

In addition to the dataset characteristics, the computational model plays another important role for prediction. Several supervised machine learning methods have been applied to build the corresponding model for balanced PPI datasets prediction, including extreme learning machine (ELM) model $[18,19]$ and support vector machines (SVM) $[15,17,20,21]$. SVM is the most utilized model in many research disciplines as it associates basic structural risk minimization theory to make predictions, while ELM model provides a faster training speed with its inherent mechanisms on Moore-Penrose generalized inverse of the neural network output. Most of these models are built for yeast and human 'intra-species' PPI.

Deep learning thrives on some benchmark datasets, for example, MNIST ${ }^{1}$, ImageNet ${ }^{2}$ and so on. Especially, deep learning has shown its powerful performance on 'Big Data' sets. This stimulates us to introduce the deep learning mechanisms into PHPPI research. Currently, more and more novel machine learning models are proposed to imitate human brain systems. Among these, CNN and DBN are two of the most popular models. In [22] the hierarchical data processing in the human brain cortex is discussed, which is imitated in the computer vision research. It has been proved in [23] that $\mathrm{CNN}$ achieves a similar data representation approach as discussed in [22]. The visualization in [23] details the construction of the corner and other edge information inside each image. Given that the models having the ability to learn higher degree features from data, deep learning is considered as a more efficient and generalized way in other research areas.

These successes in data feature representation motivate us to introduce unsupervised learning model into our study on PHPPI. In this paper, we leverage a deep stacked denoising autoencoders model to design an unsupervised learning model for PHPPI prediction. In next section, we will detail this framework and present the unsupervised learning model for PHPPI research, and later we will conduct the experiments on several curated datasets. A brief explanation of the procedure will also be presented in section IV.

\section{FRAMEWORK WITH UNSUPERVISED LEARNING FOR PHPPI}

In this section, we will first introduce and go through a whole data life cycle, which includes data curation, feature representation and model learning for PHPPI prediction.

In detail, as shown in Figure 1, this framework consists of raw data curation, data feature representation, unsupervised learning model, and eventually classifier model learning.

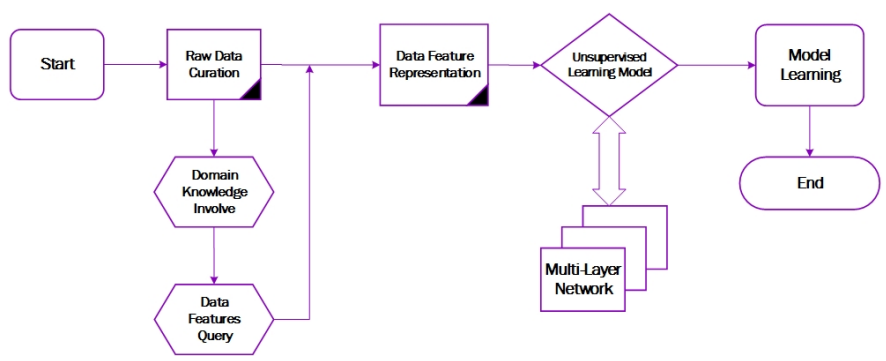

Figure 1: The Framework with Unsupervised Learning Model for PHPPI

\footnotetext{
${ }^{1}$ http://yann.lecun.com/exdb/mnist/

${ }^{2}$ http://www.image-net.org/
} 


\section{A. Raw Data Curation}

Since all positive pairs are extracted from the dispersed databases over Web, the golden dataset should contain highconfidence positive pairs and random sampling negative pairs. Normally random sampling strategy is applied between the proteins of pathogen and host to obtain the negative PHPPI pairs. In this curation phase, the setting ratio between positive and negative pairs is $1: 100$, which means one out of every 100 PHPPIs is supposed to interact with each other [11]. These protein pairs constitute the basic information of raw data. The raw data curation procedure is detailed in [24], in which data querying, data collection, data cleansing and data pairing are critical to build the raw data. Nevertheless, to further demonstrate the ability of unsupervised learning model, we also build and test datasets with $1: 25$ and $1: 50$ ratio.

\section{B. Data Feature Representation}

In the raw data curation phase, we build a dataset containing the basic information of PHPPI pairs. However the basic information only indicates the respective interacting protein ID. The ID is unique in the protein databases. The related protein feature databases to represent data into model actually include sequence information, gene ontology information, human interactome graph (since we are studying PHPPIs between human as the host and other bacterias as the pathogens) and gene expression. Correspondingly, the sequence information is stored in Uniprot [25] and gene ontology information is located in Gene Ontology Consortium [26]. The Human Protein Reference Database (HPRD) manages human interactome graph information [27]. The last one, gene expression information can be downloaded and extracted from several selected transcriptomic datasets which reflect the human genes infected by pathogens. These datasets are mostly stored in the Gene Expression Omnibus (GEO) databases [28]. A comprehensive survey on these protein features used in PHPPI study can be found in [11]

Among these protein feature databases, sequence information is the primary data feature for proteins, according to the domain knowledge: 'sequence specifies structure'. Also, because most sequences of proteins can be found in Uniprot database, in this phase we choose sequence information as our primary protein feature.

As a basis of biology knowledge, the protein is made of hundreds or even thousands of amino acids. These amino acids are organized in various lengths yet strict order to build the primary structure of different proteins. For example, a simple sequence information of protein could be: CCYGGGYYCYY. Each letter represents one of the amino acids. Basically there are four distinct structural stages which are primary structure, secondary structure, tertiary structure and finally the quaternary structure. The primary structure is mostly decided by the sequence order and length, which means the sequence information needs to be carefully processed in order to input into subsequent models. In this paper, we apply auto covariance [29] as our data feature representation method while there are some other alternative sequence representation methods as well, like conjoint triad method [30] and local descriptor [20]. It should be noted, besides the sequence information, also other features can also be further studied in the future.

The auto covariance (AC) analysis on sequence information was proposed as a good protein feature representation in [29]. Considering the numerical sequences, the auto cross covariance (ACC) is able to translate the sequence information into uniform matrices. Typically, AC considers the physicochemical properties of each amino acids in the sequence, which include hydrophobicity $(\mathrm{H})$, volumes of side chains of amino acids (VSC), polarity (P1), polarizability (P2), solvent-accessible surface area (SASA) and net charge index of side chains (NCISC). There are 20 different kinds of amino acids. Table I depicts their physiochemical properties $[11,30]$. The letter of the name is short for their respective full name, which could be found in [11].

\begin{tabular}{|l|c|c|c|c|c|c|c|}
\hline Name & H1 & H2 & Vsc & P1 & P2 & SASA & NCISC \\
\hline A & 0.62 & -0.5 & 27.5 & 8.1 & 0.046 & 1.181 & 0.007187 \\
\hline C & 0.29 & -1 & 44.6 & 5.5 & 0.128 & 1.461 & -0.03661 \\
\hline D & -0.9 & 3 & 40 & 13 & 0.105 & 1.587 & -0.02382 \\
\hline E & -0.74 & 3 & 62 & 12.3 & 0.151 & 1.862 & 0.006802 \\
\hline F & 1.19 & -2.5 & 115.5 & 5.2 & 0.29 & 2.228 & 0.037552 \\
\hline G & 0.48 & 0 & 0 & 9 & 0 & 0.881 & 0.179052 \\
\hline H & -0.4 & -0.5 & 79 & 10.4 & 0.23 & 2.025 & -0.01069 \\
\hline I & 1.38 & -1.8 & 93.5 & 5.2 & 0.186 & 1.81 & 0.021631 \\
\hline K & -1.5 & 3 & 100 & 11.3 & 0.219 & 2.258 & 0.017708 \\
\hline L & 1.06 & -1.8 & 93.5 & 4.9 & 0.186 & 1.931 & 0.051672 \\
\hline M & 0.64 & -1.3 & 94.1 & 5.7 & 0.221 & 2.034 & 0.002683 \\
\hline N & -0.78 & 2 & 58.7 & 11.6 & 0.134 & 1.655 & 0.005392 \\
\hline P & 0.12 & 0 & 41.9 & 8 & 0.131 & 1.468 & 0.239531 \\
\hline Q & -0.85 & 0.2 & 80.7 & 10.5 & 0.18 & 1.932 & 0.049211 \\
\hline R & -2.53 & 3 & 105 & 10.5 & 0.291 & 2.56 & 0.043587 \\
\hline S & -0.18 & 0.3 & 29.3 & 9.2 & 0.062 & 1.298 & 0.004627 \\
\hline T & -0.05 & -0.4 & 51.3 & 8.6 & 0.108 & 1.525 & 0.003352 \\
\hline V & 1.08 & -1.5 & 71.5 & 5.9 & 0.14 & 1.645 & 0.057004 \\
\hline W & 0.81 & -3.4 & 145.5 & 5.4 & 0.409 & 2.663 & 0.037977 \\
\hline Y & 0.26 & -2.3 & 117.3 & 6.2 & 0.298 & 2.368 & 0.023599 \\
\hline
\end{tabular}

Table I: Seven Different Physicochemical Properties for Amino Acids [29]

The AC method contains the following steps:

1) Firstly we translate sequence information into numerical values of these seven different physicochemical properties. This translation includes data normalization since the values of different properties are different in their ranges. According to Equation (1), they are all normalized to a distribution under zero mean and unit standard deviation.

$\overline{f_{i, j}}=\frac{f_{i, j}-M_{j}}{D_{j}}(i=1,2,3, \ldots, 20 ; j=1,2,3,4,5,6,7)$

2) Then we represent them into a uniform matrices by AC 
as Equation (2):

$$
A C(\text { lag }, j)=\frac{1}{N-l a g} \sum_{i=1}^{N-l a g}\left(f_{i, j}-\frac{1}{N} \sum_{i=1}^{N} f_{i, j}\right) *\left(f_{i+l a g, j}-\frac{1}{N} \sum_{i=1}^{N} f_{i, j}\right)
$$

In these formulas, $f_{i, j}$ is the $j$ th property value of $i$ th amino acid. $M_{j}$ refers to the mean value of $j$ th property over the 20 amino acids while $D_{j}$ is the standard deviation of $j$ th property over the 20 amino acids.

Based on Equation (2), a length of $l g * 7$ is calculated, where lag is the distance between two amino acids, and $l g$ is the maximum value of $l a g$.

For $m$ properties chosen out of these seven physicochemical properties, the length of the AC would be $l g * m . N$ means the length of the protein sequence.

As auto covariance is a popular transformation method for adopting numerical sequence information to uniform matrices, in this paper we also choose it as our primary data feature representation method. After AC transformation, we will concatenate each AC calculation output of the interacting proteins into one vector, in which the data feature dimensions are 420 on a pairwise level.

\section{Unsupervised Learning Model}

Deep learning has shown its superior ability on either classification or recognition problems. In this paper, we introduce the unsupervised learning model into this framework to automatically extract higher degree features for subsequent model learning.

The unsupervised learning model benefits from the sufficient amount of data. $[10,31]$ presented that the unsupervised learning model could be efficient as long as there were enough data for learning. After all, it is also a well designed model for dimensionality reduction and feature learning [32].

There are several choices for unsupervised learning model. In this paper we deploy a four-layer stacked denoising autoencoders. Similar as other research, our experiments will show that the feature extracted through unsupervised learning model, specifically the four-layer SdA, has its own advantages over the simple data feature representations.

Typically, an autoencoder is a neural network designed to be able to reconstruct the output as the same as the input. Shown in Figure 2 is a traditional autoencoder. The autoencoder tries to learn $\ddot{X} \approx X$. It contains three layers: input layer, hidden layer and output layer.

Given the input as $X=\left[x_{1}, x_{2}, \ldots, x_{n}\right]^{T}$, the hidden layer $H=\left[h_{1}, h_{2}, \ldots, h_{m}\right]$ is calculated by Equation (3):

$$
H=f\left(W * X+b_{x}\right)
$$

in which, $b_{x}$ represents the bias value, $f$ denotes the transfer function. This is the encoding calculation. To reconstruct the hidden layer, the decoding calculation is shown as Equation (4):

$$
\ddot{X}=f\left(W^{\prime} * H+b_{h}\right)
$$

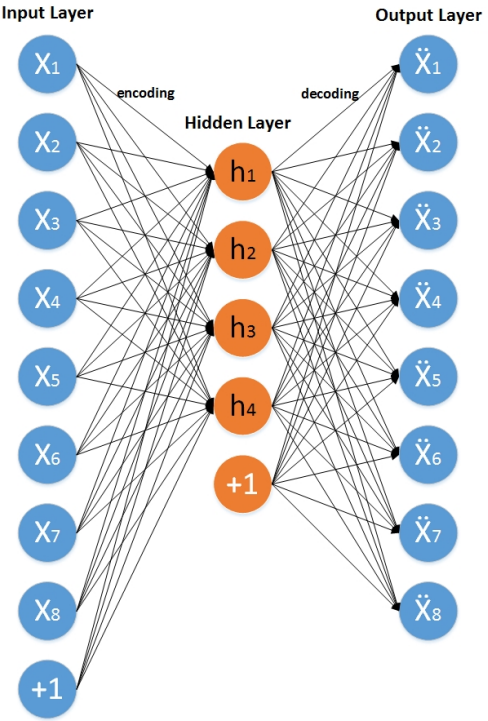

Figure 2: The Autoencoder Network

To train and measure the performance of the unsupervised learning model, one method is to calculate the mean square error (MSE) as Equation (5),

$$
\Delta=\sqrt{\frac{1}{n} \sum_{i=1}^{n}\left\|\ddot{X}_{i}-X_{i}\right\|^{2}}
$$

Denoising autoencoder, which is derived from autoencoder, tries to reconstruct an uncorrupted raw input data from a corrupted input data. The corrupted methods could be various in different scenarios. In our model, the raw input data are corrupted by adding gaussian noise.

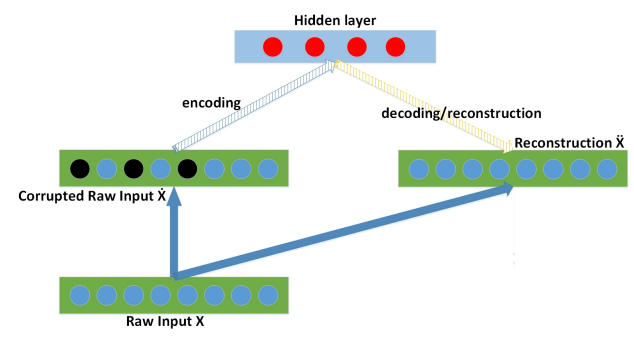

Figure 3: The Denoising Autoencoder Network

As Figure 3 shows, the $\dot{X}$ is the corrupted input data of $X$. We build the encoder by:

$$
H=f\left(W * \dot{X}+b_{x}\right)
$$

The decoding process is the same as Equation (4). By our experiments experience, the transfer function $f$ is selected as sigmoid function in our latter model for both encoding and decoding. The introduction of noise in denoising autoencoder provides this model with a better learning ability and also higher robustness. Based on denoising autoencoder 
mechanism, we build the four-layer SdA as shown in Figure 4. In this paper, it is a four-layer network model acting as our unsupervised learning model.

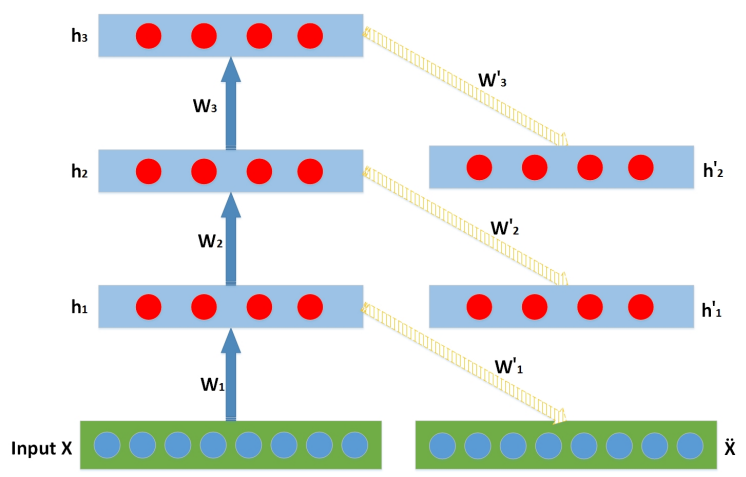

Figure 4: The Unsupervised Features Learning Network based on Stacked Denoising Autoencoder

In Figure 4, we train this $\mathrm{SdA}$ layer by layer. $h 1, h 2, h 3$ means the hidden layer outputs which we extract as our higher level features. For each denoising autoencoder, $\ddot{X} \approx$ $X, h_{i}{ }^{\prime} \approx h_{i}, i=1,2$. Later we will input $h 3$ into the classifier model for training and prediction.

\section{Classifier Model Learning}

The model learning phase is the final data learning and evaluation stage. In most cases, supervised learning models are chosen to build the final classifier learning model for further prediction. Since the datasets are relatively larger than previous balanced PPIs datasets studies, in our study, logistic regression (LR) is utilized as our supervised learning model to produce the interacting probability value between 0 and 1 .

\section{E. Summary}

In this section, we detailed our framework based on the unsupervised learning model, which could lead to an efficient performance on prediction. In section $\mathrm{V}$, we will further demonstrate that this model truly has the following superior characteristics:

- This data-driven framework achieves a better performance on PHPPI datasets. In cooperation with unsupervised learning model, we are capable of dealing with larger yet higher skewed datasets and achieve better performance.

- This framework based on unsupervised learning model, particularly with the SdA model, is an easy solution of data feature representation on PHPPI datasets. Unsupervised learning model provides better abstraction of protein sequence information and presents them to the following classifier learning model. Given several data feature representation methods, the unsupervised learning model shows its superior performance.
In the following two sections, we will elaborate our technical details and experimental results.

\section{PHPPI Datasets and Evaluation Metrics}

In this section, we will present the technical details of our study, including the statistics of the PHPPI datasets, and the evaluation metrics. The working environment is with GPU 'NVIDIA GTX970' and 64GB memory on Intel i7-6700K cpu. We tested our framework and achieved all the results on Ubuntu 14.04 system.

\section{A. PHPPI Datasets Curation}

Firstly a raw dataset is required for further data feature representation. We extracted the positive pairs from two selected PHPPI databases: PATRIC [33] and PHISTO [34]. High-confidence and experimentally verified PHPPI pairs are manually recorded and stored in these two databases. Shown as below Table II is the final statistics of the two selected bacterias.

\begin{tabular}{|l|c|c|}
\hline Bacteria Species & Positive Pairs & Ratio 1:100 \\
\hline Clostridium difficile & 52 & 5252 \\
\hline Francisella tularensis & 1338 & 135138 \\
\hline
\end{tabular}

Table II: Detail Statistics of PHPPI Datasets

Clostridium difficile and Francisella tularensis are chosen as our main pathogen species while the host remains human. Furthermore, we also conduct a comprehensive study by curating the datasets with ratio 1:25 and 1:50.

\section{B. Evaluation Metrics}

The evaluation metrics include the precision, recall value and F1 score. We still report the accuracy though in a high skewed dataset the accuracy value tells little about the model performance. Shown as below is the definition of precision and recall values:

$$
\begin{gathered}
\text { Precision }=T P /(T P+F P) \\
\text { Recall }=T P /(T P+F N)
\end{gathered}
$$

'TP', 'FP' and 'FN' are true positive number, false positive number and false negative number respectively. 'TN' refers to true negative number. F1 score is calculated by:

$$
F 1=2 * \text { Precision } /(\text { Precision }+ \text { Recall })
$$

We also draw the Receiver Operating Characteristic (ROC) curve and calculate the area value under this curve (AUC) to evaluate the performance and robustness. The ROC curve illustrates the performance of binary classifier model with the various threshold values. In this curve, the relationship between true positive rate and false positive rate can be observed.

The AUC value shows the stability and performance of a model. This value ranges from 0 to 1 . We expect the value 
to be close to 1 to achieve the best model. Regarding to the experiments settings, we present the logistic regression classifier with cross-entropy and mean squared error loss functions to learn from the Clostridium difficile dataset with 1:100 ratio. We deploy the batch gradient descent as our optimization algorithm. The batch size is 100 and learning rate equals to 0.1 . However, the logistic regression model with cross-entropy tends to predict all the output as 1 , which results in $0.01 \pm 0.0$ for accuracy and $0.5 \pm 0.0$ for AUC. Alternatively, with mean square error loss function, the model obtains $0.99 \pm 0.0$ and $0.83 \pm 0.08$ for accuracy and AUC respectively. Thus, we select mean square error as our final classifier layer loss function. These experiment settings are the same when $\mathrm{SdA}$ is added on the bottom to extract higher level representations. The experiments are carried out with 10 -fold cross-validation.

\section{RESUlt AnAlysis AND Discussion}

We train this framework on both Clostridium difficile and Francisella tularensis pathogen species. Although the expected ratio between positive and negative pairs is $1: 100$, we will also include the result on ratio of 1:25 and 1:50 which are less-imbalanced to have a comprehensive discussion.

Table III shows the precision (PR), recall (RE) and F1 score results on 1:25 ratio while Table IV is on 1:50 ratio.

\begin{tabular}{|l|c|c|}
\hline Bacteria Species & LR & SdA+LR \\
\hline \multirow{2}{*}{ Clostridium } & PR:95.28 $\pm \mathbf{9 . 4 6}$ & PR:85.78 \pm 29.78 \\
difficile & RE:87.14 $\pm \mathbf{1 4 . 9 1}$ & RE:75.71 \pm 29.31 \\
& F1:89.92 $\pm \mathbf{9 . 4 8}$ & F1:79.65 \pm 28.32 \\
\hline \multirow{2}{*}{ Francisella } & PR:96.86 $\pm \mathbf{2 . 9 1}$ & PR:93.59 \pm 6.04 \\
tularensis & RE: $15.04 \pm 1.45$ & RE:15.46 $\pm \mathbf{3 . 7 0}$ \\
& F1:26.00 \pm 2.18 & F1:26.37 $\pm \mathbf{5 . 4 3}$ \\
\hline
\end{tabular}

Table III: Evaluation Result on Ratio 1:25

\begin{tabular}{|l|c|c|}
\hline Bacteria Species & LR & SdA+LR \\
\hline \multirow{2}{*}{ Clostridium } & PR: $82.28 \pm 29.27$ & PR:98.57 $\pm \mathbf{4 . 2 9}$ \\
difficile & RE: $78.57 \pm 30.14$ & RE:90.0 $\pm \mathbf{1 4 . 3 6}$ \\
& F1: $78.82 \pm 27.26$ & F1:93.41 $\pm \mathbf{9 . 2 2}$ \\
\hline \multirow{2}{*}{ Francisella } & PR: $0 \pm 0$ & PR:98.25 $\pm \mathbf{2 . 2 1}$ \\
tularensis & RE: $0 \pm 0$ & RE:19.57 $\pm \mathbf{3 . 7 4}$ \\
& F1:0 \pm 0 & F1:32.48 $\pm \mathbf{5 . 2 0}$ \\
\hline
\end{tabular}

Table IV: Evaluation Result on Ratio 1:50

The evaluation result on dataset of 1:25 ratio shows that simple logistic regression model achieves a better performance than our unsupervised learning model. However, when the ratio becomes 1:50, which is a higher skewed ratio, our unsupervised learning model stays stable and obtains a much better result than simply supervised model learning. Figure 5 and 6 also indicate that the performance of single supervised learning model LR drops dramatically when the ratio increases from 1:25 to 1:50. We observe that the SdA model demonstrates more stable results in Figure 6. We consider that the reason for performance reduction could be related with the hyperparameters setting in LR model due to the different ratios. However, this also validates our hypothesis that stacked denoising autoencoders could be an effective model to extract higher level feature and acquire a better result in our overall study.

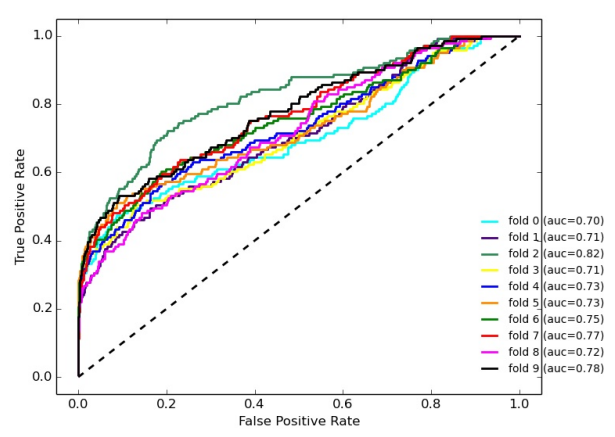

(a) Ratio 1:25

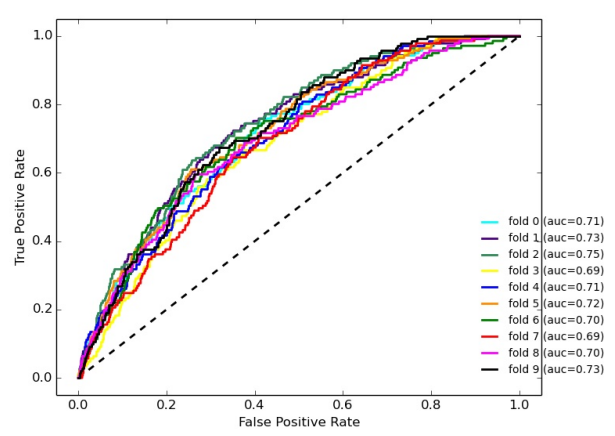

(b) Ratio 1:50

Figure 5: ROC Curve for Francisella tularensis without SdA

Though Clostridium difficile is associated with a relative smaller dataset, the high skewed ratio still introduces the uncertainty into the supervised learning model. This leads to a worse performance than our unsupervised learning model according to the results of precision and recall presented in these tables. Table $\mathrm{V}$ detail the accuracy results on the datasets with expected ratio 1:100.

\begin{tabular}{|l|c|c|}
\hline Bacteria Species & LR & SdA+LR \\
\hline Clostridium difficile & $99.00 \pm 0$ & $\mathbf{9 9 . 7 5} \pm \mathbf{0 . 2 6}$ \\
\hline Francisella tularensis & $99.00 \pm 0$ & $\mathbf{9 9 . 1 9} \pm \mathbf{0 . 0 6}$ \\
\hline
\end{tabular}

Table V: Accuracy Result on Ratio 1:100

To clarify this effect of high skewed ratio, we also present the precision, recall and F1 score on the datasets with expected ratio 1:100 in Table VI, VII and VIII respectively.

The mean value of the AUC values is tabulated in Table IX, which shows a better result with SdA. From Figures 7 to 10, we depict the ROC curves and can observe that 


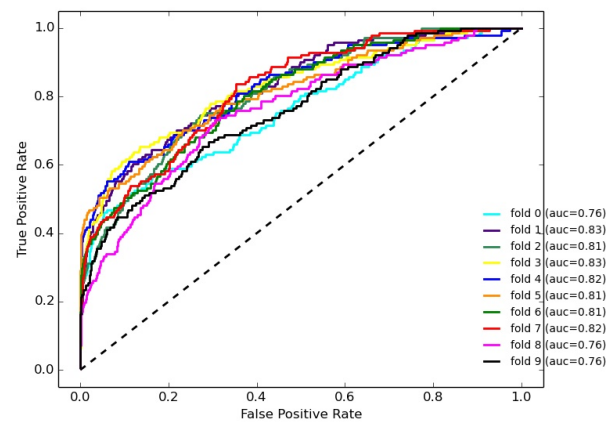

(a) Ratio 1:25

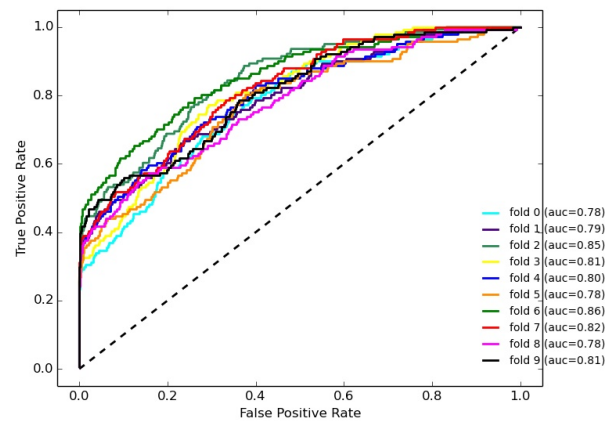

(b) Ratio 1:50

Figure 6: ROC Curve for Francisella tularensis with SdA

\begin{tabular}{|l|c|c|}
\hline Bacteria Species & LR & SdA+LR \\
\hline Clostridium difficile & $0 \pm 0$ & $\mathbf{9 8 . 7 5} \pm \mathbf{3 . 7 5}$ \\
\hline Francisella tularensis & $0 \pm 0$ & $\mathbf{9 1 . 3 4} \pm \mathbf{9 . 1 1}$ \\
\hline
\end{tabular}

Table VI: Precision Result on Ratio 1:100

\begin{tabular}{|l|c|c|}
\hline Bacteria Species & LR & SdA + LR \\
\hline Clostridium difficile & $0 \pm 0$ & $\mathbf{7 5 . 7 1} \pm \mathbf{2 7 . 1 4}$ \\
\hline Francisella tularensis & $0 \pm 0$ & $\mathbf{1 9 . 7 2} \pm \mathbf{5 . 4 8}$ \\
\hline
\end{tabular}

Table VII: Recall Result on Ratio 1:100

\begin{tabular}{|l|c|c|}
\hline Bacteria Species & LR & SdA + LR \\
\hline Clostridium difficile & $0 \pm 0$ & $\mathbf{8 2 . 2 9} \pm \mathbf{2 0 . 5 4}$ \\
\hline Francisella tularensis & $0 \pm 0$ & $\mathbf{3 2 . 1 1} \pm \mathbf{8 . 0 0}$ \\
\hline
\end{tabular}

Table VIII: F1 Score on Ratio 1:100

\begin{tabular}{|l|c|c|}
\hline Bacteria Species & LR & SdA+LR \\
\hline Clostridium difficile & $0.83 \pm 0.08$ & $\mathbf{0 . 9 6} \pm \mathbf{0 . 0 7}$ \\
\hline Francisella tularensis & $0.62 \pm 0.03$ & $\mathbf{0 . 8 2} \pm \mathbf{0 . 0 3}$ \\
\hline
\end{tabular}

Table IX: AUC Value on Ratio 1:100

the model with SdA achieves better performance for both Clostridium difficile and Francisella tularensis pathogens. The detailed curves with SdA model in these 10-fold crossvalidation experiments also show a more stable performance

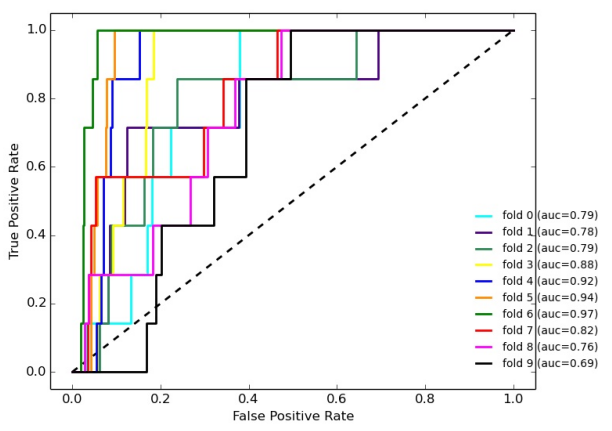

Figure 7: ROC Curve for Clostridium difficile without SdA

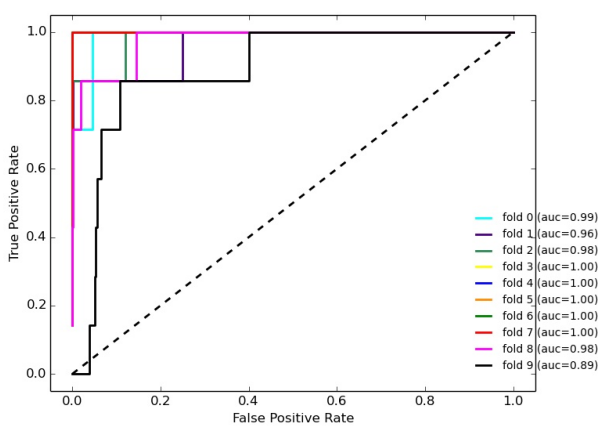

Figure 8: ROC Curve for Clostridium difficile with SdA

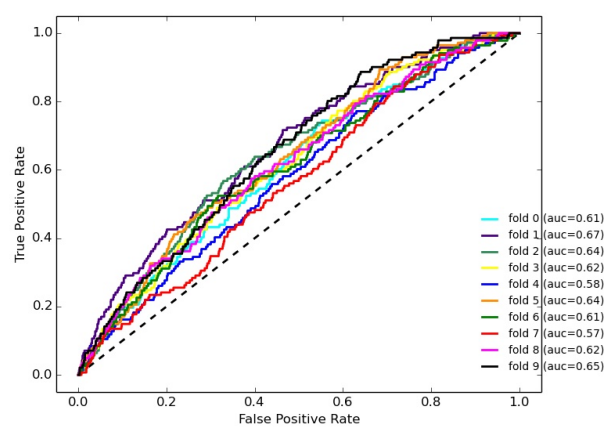

Figure 9: ROC Curve for Francisella tularensis without SdA

than single logistic regression model for PHPPI.

In this section, we presented the results to demonstrate that, unsupervised learning model, specifically with stacked denoising autoencoder, improves the model performance and obtain a better prediction results for PHPPI datasets.

\section{CONCLUSION}

In this paper, we presented a framework based on unsupervised learning model for PHPPI research. This framework benefits from higher level feature learning and achieves better performance than single classifier learning.

With the overwhelming data emerging in bioinformatics 


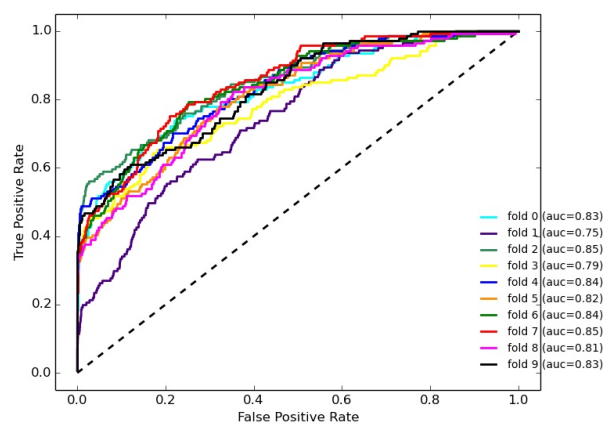

Figure 10: ROC Curve for Francisella tularensis with SdA

area, there is an upsurge of interest for high performance data analytics among the researchers. In our work, this framework has shown its superior stable ability on high skewed, imbalanced and also 'large' PHPPI datasets, while the classifier is fixed. Since PHPPI concerns about the protein-protein interactions between pathogens and hosts, this framework could deliver better insights for further multidisciplinary studies.

We also study the effect of the dataset size on PHPPI datasets. We found that unsupervised learning model could be more suitable for representing both small and big datasets. While the astonishing rate at which proteomics data is generated leads the related researches into the realm of 'Big Data', our framework in PHPPI research will be further tested with much bigger datasets in the future.

By now we have only utilized AC as our primary data feature representation method and also only used $\mathrm{SdA}$ as our unsupervised learning model. In our future work, we will concentrate on different features and introduce other unsupervised learning models into our study to further improve the model performance and its robustness, as well as applicability to other research fields.

\section{ACKNOWLEDGMENT}

This work is supported by the scholarship from the China Scholarship Council (CSC) while the first author pursues his $\mathrm{PhD}$ degree in the University of Wollongong.

\section{REFERENCES}

[1] N. Savage, "Bioinformatics: big data versus the big c," Nature, vol. 509, no. 7502, pp. S66-S67, 2014

[2] W. Raghupathi and V. Raghupathi, "Big data analytics in healthcare: promise and potential," Health Information Science and Systems, vol. 2, no. 1, p. 3, 2014.

[3] G. Sun, T. Cui, G. Beydoun, J. Shen, and S. Chen, "Profiling and supporting adaptive micro learning on open education resources," in Advanced Cloud and Big Data(CBD), 2016 International Conference on, IEEE, 2016, pp. 158-163.

[4] C. S. Greene, J. Tan, M. Ung, J. H. Moore, and C. Cheng, "Big data bioinformatics," Journal of cellular physiology, vol. 229, no. 12, pp. 1896-1900, 2014.

[5] V. Marx, "Biology: The big challenges of big data," Nature, vol. 498, no. 7453, pp. $255-260,2013$

[6] M. D. Ritchie, E. R. Holzinger, R. Li, S. A. Pendergrass, and D. Kim, "Methods of integrating data to uncover genotypephenotype interactions," Nat. Rev. Genet., vol. 16, no. 2, pp. 85-97, 2015

[7] S. Min, B. Lee, and S. Yoon, "Deep learning in bioinformatics," Briefings in Bioinformatics, p. bbw068, 2016.

[8] G. E. Hinton, S. Osindero, and Y.-W. Teh, "A fast learning algorithm for deep belief nets," Neural computation, vol. 18, no. 7, pp. 1527-1554, 2006.
[9] G. E. Hinton and R. R. Salakhutdinov, "Reducing the dimensionality of data with neural networks," science, vol. 313, no. 5786, pp. 504-507, 2006

[10] Y. LeCun, Y. Bengio, and G. Hinton, "Deep learning," Nature, vol. 521, no. 7553, pp. 436-444, 2015

[11] H. Chen, J. Shen, L. Wang, and J. Song, "Towards data analytics of pathogenhost protein-protein interaction: a survey," in Big Data(BigData Congress), 2016 IEEE International Congress on, IEEE, 2016, pp. 377-388.

[12] O. Krishnadev and N. Srinivasan, "Prediction of protein-protein interactions between human host and a pathogen and its application to three pathogenic bacteria." Int. J. Biol. Macromol., vol. 48, no. 4, pp. 613-619, 2011.

[13] T. Ito, T. Chiba, R. Ozawa, M. Yoshida, M. Hattori, and Y. Sakaki, "A comprehensive two-hybrid analysis to explore the yeast protein interactome," Proceedings of the National Academy of Sciences, vol. 98, no. 8, pp. 45694574, 2001.

[14] Q. C. Zhang, D. Petrey, L. Deng, L. Qiang, Y. Shi, C. A. Thu, B. Bisikirska, C. Lefebvre, D. Accili, T. Hunter, T. Maniatis, A. Califano, and B. Honig, "Structure-based prediction of proteinprotein interactions on a genome-wide scale," Nature, vol. 490, no. 7421, pp. 556-560, 2012.

[15] Z. You, Z. Ming, B. Niu, S. Deng, and Z. Zhu, "A svm-based system for predicting protein-protein interactions using a novel representation of protein sequences," in Intelligent Computing Theories. Springer, 2013, pp. 629-637.

[16] M. Kshirsagar, J. Carbonell, and J. Klein-Seetharaman, "Techniques to cope with missing data in host-pathogen protein interaction prediction," Bioinformatics, vol. 28 , no. 18, pp. 466-472, 2012

[17] — " "Multisource transfer learning for host-pathogen protein interaction prediction in unlabeled tasks," NIPS Work. Mach. Learn. Comput. Biol., no. 1, pp. 3-6, 2013.

[18] Z.-H. You, Y.-K. Lei, L. Zhu, J. Xia, and B. Wang, "Prediction of proteinprotein interactions from amino acid sequences with ensemble extreme learning machines and principal component analysis," BMC Bioinformatics, vol. 14, no. Suppl 8, p. S10, 2013.

[19] Z.-H. You, S. Li, X. Gao, X. Luo, and Z. Ji, "Large-scale protein-protein interactions detection by integrating big biosensing data with computational model." Biomed Res. Int., vol. 2014, p. 598129, 2014.

[20] M. N. Davies, A. Secker, A. A. Freitas, E. Clark, J. Timmis, and D. R. Flower, "Optimizing amino acid groupings for gpcr classification," Bioinformatics, vol. 24, no. 18, pp. 1980-1986, 2008.

[21] G. Cui, C. Fang, and K. Han, "Prediction of protein-protein interactions between viruses and human by an svm model," BMC bioinformatics, vol. 13, no. 7, p. 1, 2012.

[22] N. Kruger, P. Janssen, S. Kalkan, M. Lappe, A. Leonardis, J. Piater, A. J. Rodriguez-Sanchez, and L. Wiskott, "Deep hierarchies in the primate visual cortex: What can we learn for computer vision?" IEEE transactions on pattern analysis and machine intelligence, vol. 35, no. 8, pp. 1847-1871, 2013.

[23] M. D. Zeiler and R. Fergus, "Visualizing and understanding convolutional networks," in European conference on computer vision, Springer, 2014, pp. 818833.

[24] H. Chen, J. Shen, L. Wang, and J. Song, "Collaborative data analytics towards prediction on pathogen-host protein-protein interactions," in Computer Supported Cooperative Work in Design(CSCWD), 2017 IEEE 21st International Conference on, IEEE, 2017, to appear.

[25] U. Consortium et al., "Uniprot: a hub for protein information," Nucleic acids research, p. gku989, 2014

[26] G. O. Consortium et al., "Gene ontology consortium: going forward," Nucleic acids research, vol. 43, no. D1, pp. D1049-D1056, 2015.

[27] R. Goel, H. Harsha, A. Pandey, and T. K. Prasad, "Human protein reference database and human proteinpedia as resources for phosphoproteome analysis," Molecular bioSystems, vol. 8, no. 2, pp. 453-463, 2012

[28] T. Barrett, S. E. Wilhite, P. Ledoux, C. Evangelista, I. F. Kim, M. Tomashevsky, K. A. Marshall, K. H. Phillippy, P. M. Sherman, M. Holko et al., "Ncbi geo: archive for functional genomics data setsupdate," Nucleic acids research, vol. 41 , no. D1, pp. D991-D995, 2013

[29] Y. Guo, L. Yu, Z. Wen, and M. Li, "Using support vector machine combined with auto covariance to predict protein-protein interactions from protein sequences," Nucleic acids research, vol. 36, no. 9, pp. 3025-3030, 2008.

[30] J. Shen, J. Zhang, X. Luo, W. Zhu, K. Yu, K. Chen, Y. Li, and H. Jiang, "Predicting protein-protein interactions based only on sequences information," Proc. Natl. Acad. Sci., vol. 104, no. 11, pp. 4337-4341, 2007.

[31] P. Vincent, H. Larochelle, Y. Bengio, and P.-A. Manzagol, "Extracting and composing robust features with denoising autoencoders," in Proceedings of the 25th international conference on Machine learning, ACM, 2008, pp. 1096-1103.

[32] I. Goodfellow, Y. Bengio, and A. Courville, Deep learning, MIT Press, 2016.

[33] A. R. Wattam, D. Abraham, O. Dalay, T. L. Disz, T. Driscoll, J. L. Gabbard, J. J. Gillespie, R. Gough, D. Hix, R. Kenyon et al., "Patric, the bacteria bioinformatics database and analysis resource," Nucleic acids research, p. gkt1099, 2013

[34] S. D. Tekir, T. Çakır, E. Ardıç, A. S. Sayılırbaş, G. Konuk, M. Konuk, H. Sarıyer, A. Uğurlu, İ. Karadeniz, A. Özgür et al., "Phisto: pathogen-host interaction search tool," Bioinformatics, vol. 29, no. 10, pp. 1357-1358, 2013. 